\title{
Blue phases decorated with colloidal particles: photonic crystals
}

\author{
Miha Ravnik ${ }^{1,2}$ and Slobodan Zumer ${ }^{1,3,4}$ \\ ${ }^{I}$ Faculty of Mathematics and Physics, University of Ljubljana, Slovenia \\ ${ }^{2}$ Rudolf Peierls Centre for Theoretical Physics, University of Oxford, UK \\ ${ }^{3}$ Jozef Stefan Institute, Slovenia \\ ${ }^{4}$ Center of Excellence NAMASTE, Slovenia
}

Received February 21, 2011; accepted March 11, 2011; published March 31, 2011

\begin{abstract}
The recent efforts to understand self-assembly of 'soft' photonic colloidal crystals in intrinsic disclination networks of body centered and face centered cubic lattices of blue phases I and II are briefly reviewed.
\end{abstract}

Soft photonic crystals that could be easily manipulated via external stimuli and that could be formed through self-assembly would be of great interest for photonic and plasmonic applications [1]. One of the perspective templates for such materials are blue phase crystals where chiral nematic fluid exhibits periodic 3D modulation of the average molecular orientation [2]. These structures have cubic network of orthogonal double-twist cylinders intercalated by a network of $-1 / 2$ disclination lines and are the result of the frustration occurring when space is filled with intrinsically preferred double twist nematic director configurations [2]. Best-known are blue phase I (BP I) with body centered cubic unit cell and blue phase II (BP II) with a simple cubic cell (see Fig. 1).
The applications of these structures, like electrically controlled lasing [3] and large electro-optic Kerr effect [4], were until recently very limited by the narrow $(\sim 1 \mathrm{~K})$ temperature stability region. Recent developments that introduce blue phases with an extended stability range [47] make them more attractive for applications. These advances stimulated us to apply modeling and simulations to examine the possibility of using blue phases as templates for assembling 3D colloidal crystals. Here we briefly review our recent achievements [8-10].

The superstructures of disclination lines act as natural templates for assembling colloidal crystals. Each disclination adds to the elastic energy of the system. Introducing particles that generate their own distortions leads to further increase unless they are arranged and get trapped in the disclination line. This general thermodynamic principle yields effective potentials that drive the trapping of colloidal particles into disclination lines (see Fig. 2).

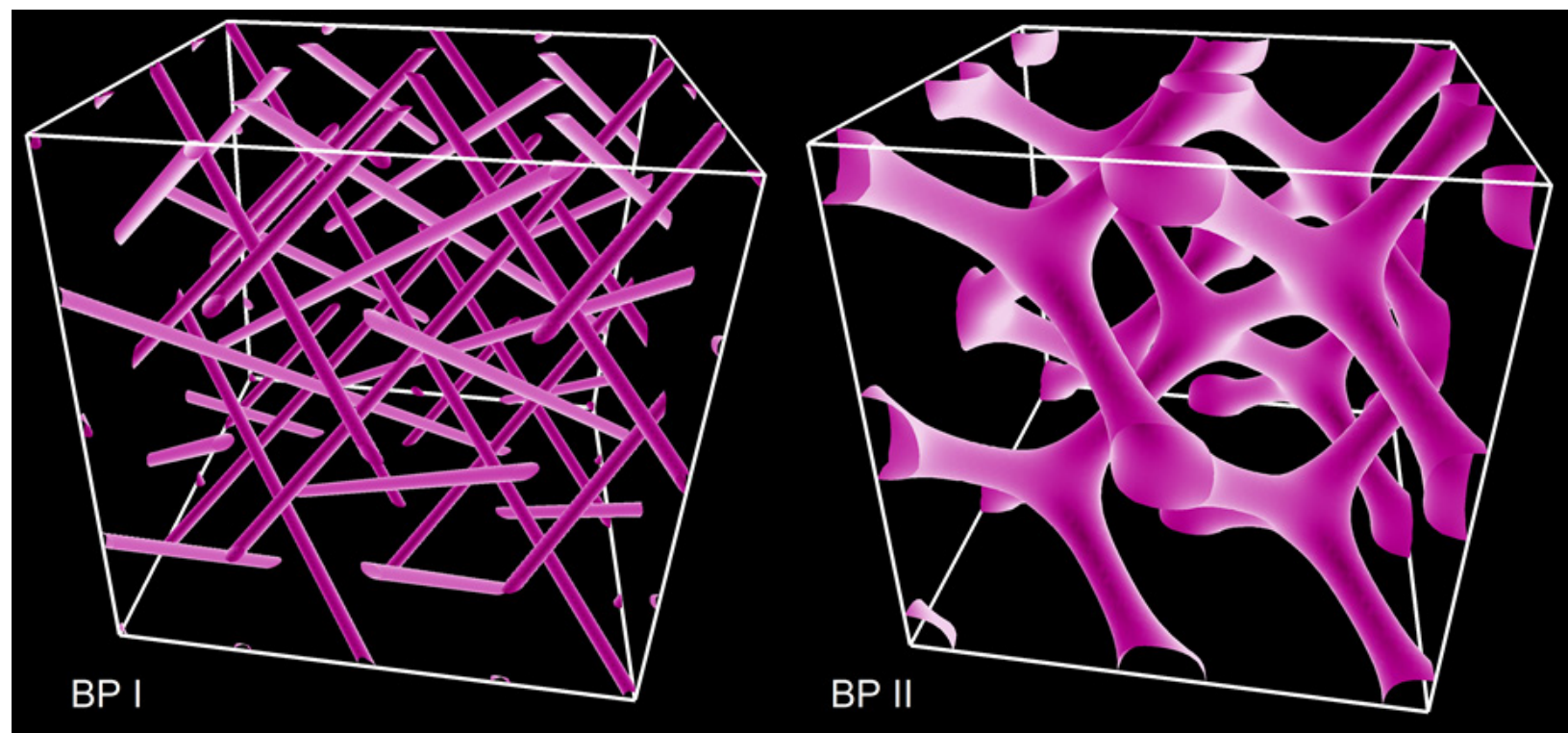

Fig. 1. Arrays of disclination lines in blue phase I (BP I) and blue phase II (BP II). Disclinations are visualized as iso-surfaces of degree of order $\mathrm{S}=0.3$ in BP I and S=0.17 in BP II. Director fields characterized by arrays of double twist cylinders [2] are not shown. Boxes constituted of $2 \times 2 \times 2$ unit cells are shown (one unit cell length is $360 \mathrm{~nm}$ in BP I and $680 \mathrm{~nm}$ in BP II). 


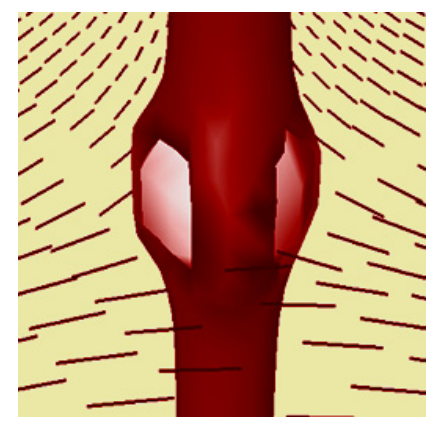

Fig. 2. Trapping of a small 30nm sized homeotropic particle by a $-1 / 2$ disclination line in a non-chiral nematic. Defect structure is shown as iso-surface of degree of order $S=0.5$.

In our earlier study of hierarchal structures we show how small colloidal particles self -assemble in $-1 / 2$ disclination lines that entangle large colloidal particles [11]. For particle sizes of a few $100 \mathrm{~nm}$, the effective trapping potential, i.e. the changes in the free energy, can be as high as a few 100kT. It should be stressed that the trapping potential is strongly anisotropic, yielding alternating three attractive and three repulsive directions for both particles with hometoropic and planar anchoring.
Our numerical studies are mostly based on a Landau-de Gennes phenomenological description and nematic orientational order tensor. The free energy includes phase contribution written in terms of the powers of the order parameter, gradient term with included chiral contribution, and surface term describing anchoring phenomenon. For details see Ref. [12]. We show that the disclination network can be used to trap colloidal particles and also allow for a partial self-assembly of 3D colloidal crystals [810]. Further, we show how the particle size ranging from nano- to micro-size affects the stability of 3D colloidal crystals. Particularly relevant are surface anchoring and particle size. The regular distribution of particles adopts the cubic symmetry of the array of disclination lines with details depending on specific particle positions.

Figure 3 shows two stable 3D blue phase colloidal crystals, with different filling fractions. The equilibrium positions of spherical particles, indeed, clearly coincide with the cores of the disclination lines. This allows particles to replace the maximum part of the energetically unfavorable region where the order region where the order is strongly distorted with their own volume. For details, in particular the discussion about the optimal size of the colloidal particles, see Ref. [10].

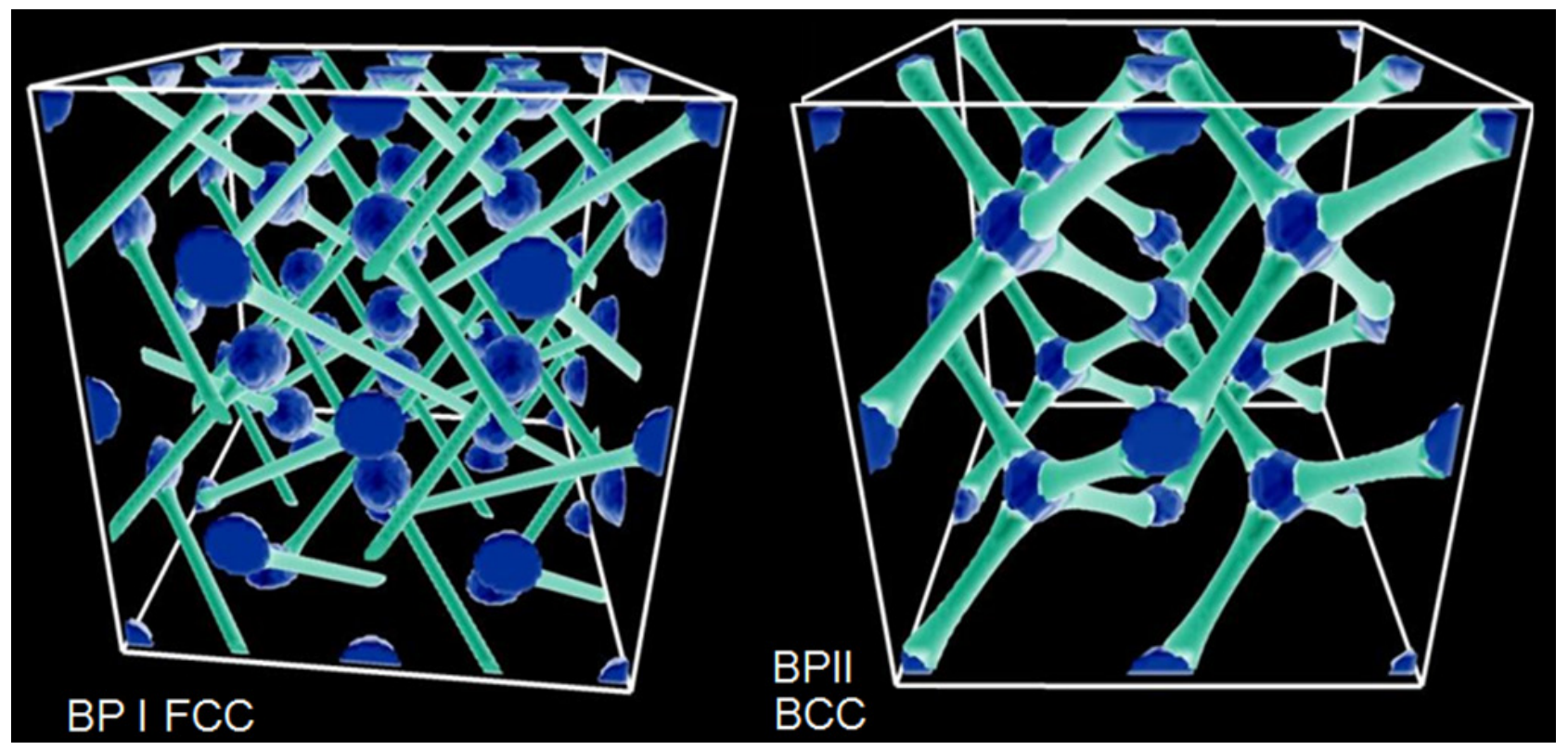

Fig. 3. BP colloidal crystals $(2 \times 2 \times 2$ unit cells): BP I FCC colloidal crystal with 4 particles per unit cell, and BP II BCC colloidal crystal with 2 particles per unit cell. In BP I disclination lines are drawn as iso-surfaces of degree of order $\mathrm{S}=0.23\left(\mathrm{~S}_{\text {bulk }}=0.40\right)$ with particle radius $70 \mathrm{~nm}$. In BP II disclination lines are visualized as isosurfaces of $\mathrm{S}=0.1\left(\mathrm{~S}_{\text {bulk }}=0.26\right)$ with particle radius $50 \mathrm{~nm}$. 
The reduction in free energy due to the trapping of particles in disclination lines directly increases the thermodynamic stability range of both blue phase I and II. In Fig. 4 we reproduce the phase diagram of colloidal blue phases, calculated numerically for spherical particles with a $100 \mathrm{~nm}$ radius and weak homeotropic anchoring $\left(W=10^{-5} \mathrm{~J} / \mathrm{m}^{2}\right)$. For a short pitch the stability temperature range increases even for factor $\sim 3$.

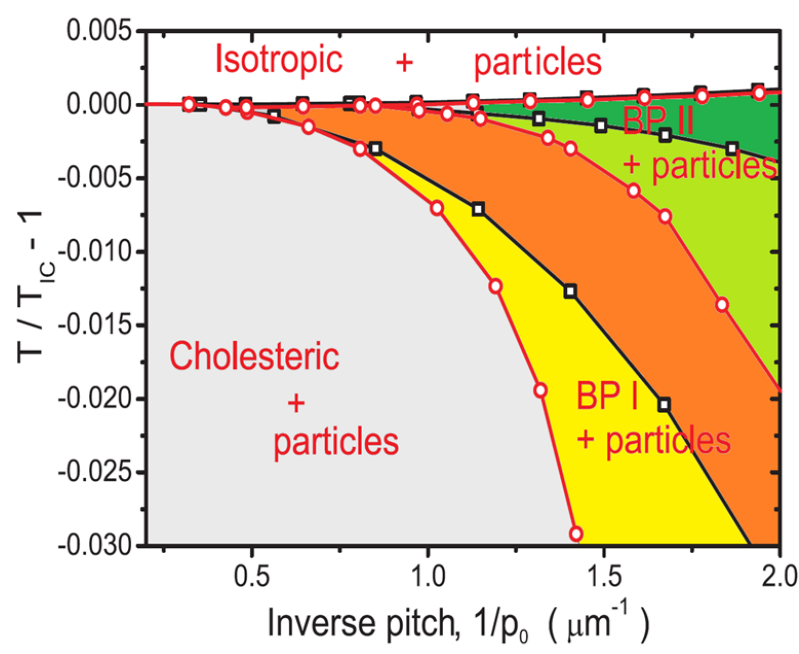

Fig. 4. Effect of colloidal particles (100 nm radius and weak homeotropic anchoring) trapped in a BP disclination line on the stability of phases. Stability regions (isotropic, BP I, BP II, and cholesteric) are plotted in terms of temperature and inverse pitch. The yellow and light green areas are the expanded stability ranges of BP I with 4 particles per unit cell and BP II with 2 particles, respectively.
We believe that the modeled particle assemblies in the blue phases can be realized also experimentally, producing soft photonic crystals that are inherently highly susceptible to manipulation via affecting the liquid crystal matrix and/or functionalized colloidal particles. This intrinsic tunability of blue phase colloidal materials could, in future, allow us to selfassemble complex structures for use in photonics and plasmonics.

MR acknowledges the support of the EC under the Marie Curie Programme ACTOIDS; MR and SZ acknowledge funding from ARRS P1-0099, J12335, FP7 215851-2 HIERARCHY.

\section{References}

[1] S. Wong, V. Kitaev, G.A. Ozin, J. Am. Chem. Soc. 125, 15589 (2003).

[2] D.C. Wright, N.D. Mermin, Rev. Mod. Phys. 61, 385 (1989).

[3] W. Cao, A. Munoz, P. Palffy-Muhoray, and B. Taheri, Nature Mater. 1, 111 (2002)

[4] Y. Hisakado, H. Kikuchi, T. Nagamura, T. Kajiyama, Adv. Mater. 17, 96 (2005)

[5] H. Kikuchi, M. Yokota, Y. Hisakado, H. Yang, T. Kajiyama, Nature Mater. 1, 64 (2002).

[6] H.J. Coles, M.N. Pivnenko, Nature 436, 997 (2005).

[7] F. Castles, S.M. Morris, E.M. Terentjev, H.J. Coles, Phys. Rev. Lett 104, 157801 (2010).

[8] M. Ravnik, G.P. Alexander, J.M. Yeomans, S. Zumer, Faraday Discuss. 144, 159 (2010).

[9] S. Zumer, M. Ravnik, T. Porenta, G.P. Alexander, J.M. Yeomans, Proc. SPIE 7775, 77750H (2010).

[10] M. Ravnik, G.P. Alexander, J.M. Yeomans, S. Zumer, PNAS Early Edition, February 28, 2011; doi:10.1073/pnas.1015831108.

[11] M. Skarabot, M. Ravnik, S. Zumer, U. Tkalec, I. Poberaj, D. Babic, I. Musevic, Phys. Rev. E 77, 061706 (2008).

[12] M. Ravnik, S. Zumer, Liq. Cryst. 36, 1201 (2009). 